The concept of bias is the lack of internal validity or incorrect assessment of the association between an exposure and an effect in the target population in which the statistic estimated has an expectation that does not equal the true value. Biases can be classified by the research stage in which they occur or by the direction of change in a estimate. The most important biases are those produced in the definition and selection of the study population, data collection, and the association between different determinants of an effect in the population. A definition of the most common biases occurring in these stages is given.

See end of article for authors' affiliations

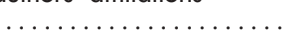

Correspondence to: Professor M DelgadoRodríguez, Division of Preventive Medicine and Public Health, Building B-3, University of Jaen, 23071-Jaén, Spain: mdelgado@ujaen.es

Accepted for publication 15 November 2003
$\mathrm{T}$ he concept of bias is the lack of internal validity or incorrect assessment of the association between an exposure and an effect in the target population. In contrast, external validity conveys the meaning of generalisation of the results observed in one population to others. There is not external validity without internal validity, but the presence of the second does not guarantee the first. Bias should be distinguished from random error or lack of precision. Sometimes, the term bias is also used to refer to the mechanism that produces lack of internal validity. ${ }^{1}$

Biases can be classified by the direction of the change they produce in a parameter (for example, the odds ratio (OR)). Toward the null bias or negative bias yields estimates closer to the null value (for example, lower and closer OR to 1), whereas away from the null bias produces the opposite, higher estimates than the true ones. An exaggeration of these biases can induce a switchover bias, or change of the direction of association (for example, a true OR $>1$ becomes $<1$ ). ${ }^{2}$

There are several classifications of bias. Sackett ${ }^{3}$ and $\mathrm{Choi}^{4}$ classified biases according to the stages of research that can occur: reading up on the field, specification and selection the study sample, execution of the experimental manoeuvre, measurement of exposures/outcomes, data analysis, results interpretation and publication. Maclure and Schneeweiss, ${ }^{5}$ applying the causal diagram theory, offered an interesting explanation of the main sources of bias. Kleinbaum et al, ${ }^{2}$ based on Olli S Miettinen's ideas, classified biases in three main groups: selection bias, information bias, and confounding. Steineck and Ahlbom, ${ }^{6}$ based on the Miettinen's concept of study base, considered in this order, confounding, misclassification (similar to information bias), misrepresentation (which has a narrower meaning than selection bias), and analysis deviation. Steineck and
Ahlbom keep confounding apart from biases in the statistical analysis as it typically occurs when the actual study base differs from the "ideal" study base, in which there is no association between different determinants of an effect. The same idea can be found in Maclure and Schneeweiss. ${ }^{5}$

In this glossary definitions of the most common biases (we have not been exhaustive in defining all the existing biases) are given within the simple classification by Kleinbaum et al. ${ }^{2} \mathrm{We}$ have added a point for biases produced in a trial in the execution of the intervention. Biases in data interpretation, writing, and citing will not be discussed (see for a description of them by Sackett ${ }^{3}$ and $\mathrm{Choi}^{4}$ ). Biases in data analysis are very numerous, but they are easily solved using appropriate procedures; they are not commented on in this glossary, unless a particular bias has an additional influence. This occurs with post hoc analysis that may lead to a publication bias when significant results are more frequently reported. Confounding bias is kept apart from biases in data analysis (according to the ideas of Steineck and Ahlbom ${ }^{6}$ and Maclure and Schneeweiss ${ }^{5}$ ).

Table 1 gives an alphabetical list of biases. The type of bias and the design affected is also given.

\section{SELECTION BIAS}

The error introduced when the study population does not represent the target population..$^{78}$ Selection bias can be controlled when the variables influencing selection are measured on all study subjects and either (a) they are antecedents of both exposure and outcome or (b) the joint distribution of these variables (plus exposure and outcome) is known in the whole target population, or (c) the selection probabilities for each level of these variables are known. ${ }^{9}$ It can be introduced at any stage of a research study ${ }^{7}$ : design (bad definition of the eligible population, lack of accuracy of sampling frame, uneven diagnostic procedures in the target population) and implementation.

\subsection{Inappropriate definition of the eligible population}

In any kind of design ascertainment bias can occur. It is produced when the kind of patients gathered does not represent the cases originated in the population $^{4}$ (see Pollock et $a l^{10}$ for an illustration). It may be produced, among many possibilities, by healthcare access bias, length-biased sampling, Neyman bias, competing risks, or survivor treatment selection bias. In studies on evaluation of a diagnostic test the spectrum bias is a kind of ascertainment bias. The definitions of these biases in alphabetical order are the following: 
Table 1 Alphabetical list of biases, indicating their type and the design where they can occur

\begin{tabular}{|c|c|c|c|}
\hline Specific name of bias & Group of bias & $\begin{array}{l}\text { Subgroup of bias (next } \\
\text { level to specific name) }\end{array}$ & Type of design affected \\
\hline Allocation of intervention bias & Execution of an intervention & & Trial \\
\hline Apprehension bias & Information bias & Observer bias & All studies \\
\hline Ascertainment bias & Selection bias & $\begin{array}{l}\text { Inappropriate definition of the } \\
\text { eligible population }\end{array}$ & Observational study \\
\hline Berkson's bias & Selection bias & $\begin{array}{l}\text { Inappropriate definition of the } \\
\text { eligible population }\end{array}$ & Hospital based case-control study \\
\hline Centripetal bias & Selection bias & Healthcare access bias & Observational study \\
\hline Citation bias & Selection bias & $\begin{array}{l}\text { Lack of accuracy of sampling } \\
\text { frame }\end{array}$ & Systematic review/meta-analysis \\
\hline Competing risks & Selection bias & Ascertainment bias & All studies \\
\hline Compliance bias & Execution of an intervention & & Trial \\
\hline Confounding by group & Confounding & & Ecological study \\
\hline Confounding by indication & Confounding & & Case-control study, cohort study \\
\hline Contamination bias & Execution of an intervention & & Trial, mainly community trials \\
\hline Detection bias & Selection bias & $\begin{array}{l}\text { Uneven diagnostic procedures } \\
\text { in the target population }\end{array}$ & Case-control study \\
\hline Detection bias & Information bias & Misclassification bias & Cohort study \\
\hline Diagnostic/treatment access bias & Selection bias & Healthcare access bias & Observational study \\
\hline Diagnostic suspicion bias & Selection bias & Detection bias & Case-control study \\
\hline Diagnostic suspicion bias & Information bias & Detection bias & Cohort study \\
\hline Differential maturing & & & Trial \\
\hline Differential misclassification bias & Information bias & Misclassification bias & All studies \\
\hline Dissemination bias & Selection bias & $\begin{array}{l}\text { Lack of accuracy of sampling } \\
\text { frame }\end{array}$ & Systematic review/meta-analysis \\
\hline Ecological fallacy & Information bias & & Ecological study \\
\hline Exclusion bias & Selection bias & $\begin{array}{l}\text { Inappropriate definition of the } \\
\text { eligible population }\end{array}$ & Case-control study \\
\hline Exposure suspicion bias & Information bias & Recall bias & Case-control study \\
\hline Family aggregation bias & Information bias & Reporting bias & Observational study \\
\hline Friend control bias & Selection bias & $\begin{array}{l}\text { Inappropriate definition of the } \\
\text { eligible population }\end{array}$ & Case-control study \\
\hline Hawthorne effect & Information bias & & Trial \\
\hline Healthcare access bias & Selection bias & Ascertainment bias & Observational study \\
\hline Healthy volunteer bias & Selection bias & Non-response bias & Observational study \\
\hline Healthy worker effect & Selection bias & $\begin{array}{l}\text { Inappropriate definition of the } \\
\text { eligible population }\end{array}$ & $\begin{array}{l}\text { Cohort study (mainly } \\
\text { retrospective) }\end{array}$ \\
\hline \multicolumn{4}{|c|}{ Incidence-prevalence bias (synonym of Neyman bias) } \\
\hline Inclusion bias & Selection bias & $\begin{array}{l}\text { Inappropriate definition of the } \\
\text { eligible population }\end{array}$ & Hospital based case-control study \\
\hline Lack of intention to treat analysis & & & Randomised trial \\
\hline Language bias & Selection bias & $\begin{array}{l}\text { Inappropriate definition of the } \\
\text { eligible population }\end{array}$ & Systematic review/meta-analysis \\
\hline Lead-time bias & Information bias & & Screening study \\
\hline Length biased sampling & Selection bias & Ascertainment bias & Cross sectional study, screening \\
\hline Losses/withdrawals to follow up & Selection bias & During study implementation & Cohort study, trial \\
\hline Mimicry bias & Selection bias & Detection bias & Case-control study \\
\hline Mimicry bias & Information bias & Detection bias & Cohort study \\
\hline Misclassification bias & Information bias & & All studies \\
\hline Missing information in multivariable analysis & Selection bias & During study implementation & All studies (mainly retrospective) \\
\hline Mode for mean bias & Information bias & Reporting bias & All studies \\
\hline Neyman bias & Selection bias & Ascertainment bias & $\begin{array}{l}\text { Cross sectional study, case-control } \\
\text { study with prevalent cases }\end{array}$ \\
\hline Non-differential misclassification bias & Information bias & Misclassification bias & All studies \\
\hline Non-random sampling bias & Selection bias & $\begin{array}{l}\text { Lack of accuracy of sampling } \\
\text { frame }\end{array}$ & Observational study \\
\hline Non-response bias & Selection bias & During study implementation & Observational study \\
\hline Obsequiousness bias & Information bias & Reporting bias & All studies \\
\hline Observer expectation bias & Information bias & Observer bias & All studies \\
\hline Observer/interviewer bias & Information bias & Misclassification bias & All studies \\
\hline Overmatching & Selection bias & $\begin{array}{l}\text { Inappropriate definition of the } \\
\text { eligible population }\end{array}$ & Case-control study \\
\hline Participant expectation bias & Information bias & Recall bias & Trial \\
\hline Popularity bias & Selection bias & Healthcare access bias & Observational study \\
\hline Post hoc analysis & Selection bias & Publication bias & Systematic review/meta-analysis \\
\hline Protopathic bias & Information bias & & Observational study \\
\hline Publication bias & Selection bias & $\begin{array}{l}\text { Lack of accuracy of sampling } \\
\text { frame }\end{array}$ & Systematic review/meta-analysis \\
\hline Purity diagnostic bias & Selection bias & Spectrum bias & Validity of diagnostic tests \\
\hline Recall bias & Information bias & Misclassification bias & All studies \\
\hline Referral filter bias & Selection bias & Healthcare access bias & Observational study \\
\hline Regression dilution bias & Information bias & Regression to the mean & Cohort study, trial \\
\hline Regression to the mean & Information bias & & Cohort study, trial \\
\hline Relative control bias & Selection bias & $\begin{array}{l}\text { Inappropriate definition of the } \\
\text { eligible population }\end{array}$ & Case-control study \\
\hline Reporting bias & Information bias & Misclassification bias & All studies \\
\hline Rumination bias & Information bias & Recall bias & $\begin{array}{l}\text { Case-control study, retrospective } \\
\text { cohort study }\end{array}$ \\
\hline
\end{tabular}


Table 1 Continued

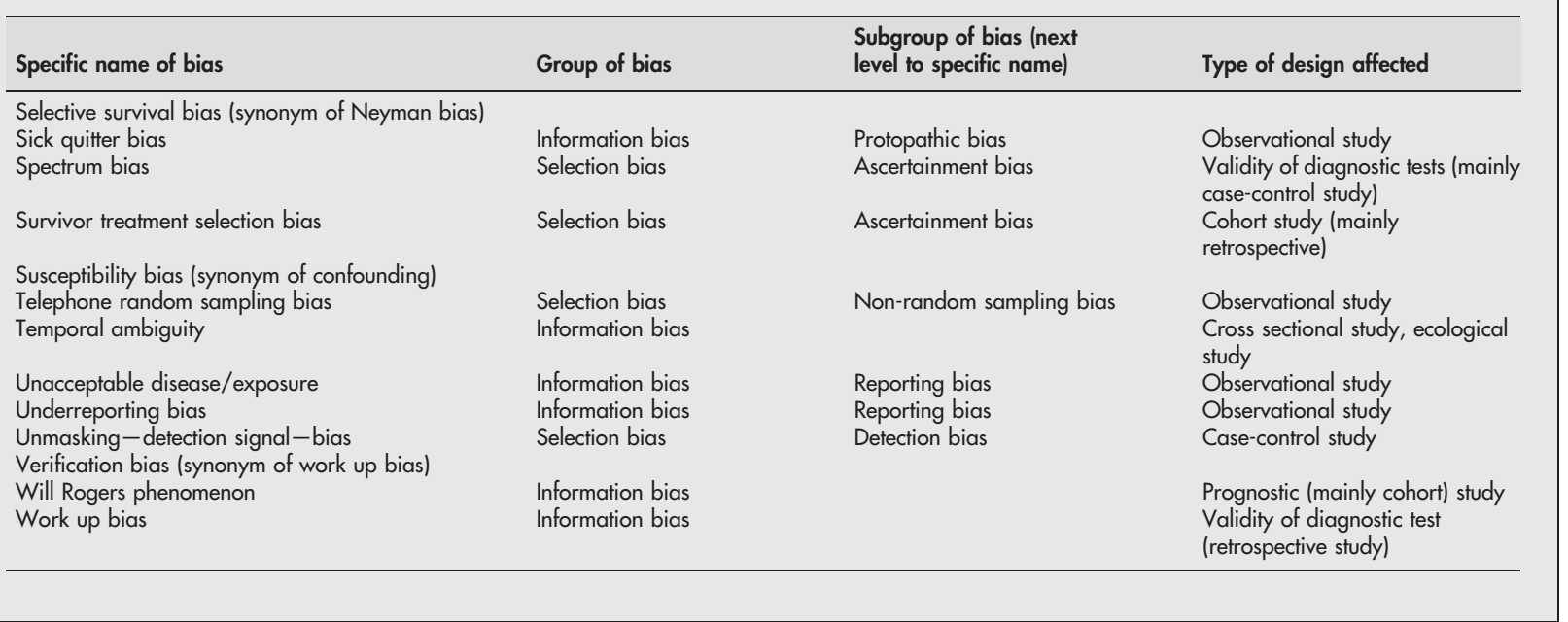

- Competing risks: when two or more outputs are mutually exclusive, any of them competes with each other in the same subject. It is more frequent when dealing with causes of death: as any person only dies once, the risk for a specific cause of death can be affected by an earlier one. For example, early death by AIDS can produce a decrease in liver failure mortality in parenteral drug users. A proper analysis of this question should take into account the competing causes of death; for instance, estimating the probability of death by a specific cause of death if any other risk of death is removed (the so called net probability of death). ${ }^{11} 12$

- Healthcare access bias: when the patients admitted to an institution do not represent the cases originated in the community. This may be due: to the own institution if admission is determined by the interest of health personnel on certain kind of cases (popularity bias), ${ }^{3}$ to the patients if they are attracted by the prestige of certain clinicians (centripetal bias), ${ }^{3}$ to the healthcare organisation if it is organised in increasing levels of complexity (primary, secondary, and tertiary care) and "difficult" cases are referred to tertiary care (referral filter bias), ${ }^{3}$ to a web of causes if patients by cultural, geographical, or economic reasons show a differential degree of access to an institution (diagnostic/treatment access bias). ${ }^{3}$

- Length-bias sampling: cases with diseases with long duration are more easily included in surveys. This series may not represent the cases originated in the target population. ${ }^{13}$ These cases usually have a better prognosis.

- Neyman bias: (synonyms: incidence-prevalence bias, selective survival bias) when a series of survivors is selected, if the exposure is related to prognostic factors, or the exposure itself is a prognostic determinant, the sample of cases offers a distorted frequency of the exposure. ${ }^{14}$ This bias can occur in both cross sectional and (prevalent) case-control studies. Lets suppose that a case-control study is carried out to study the relation between tobacco smoking and acute myocardial infarction (AMI), being cases interviewed one week after the coronary attack. If smoker patients with AMI die more frequently, the leaving cases will show lower frequency of smoking, undervaluing the association between smoking and AMI. It has been shown that the bias occurs only if the risk factor influences mortality from the disease being studied. ${ }^{15}$

- Spectrum bias: in the assessment of validity of a diagnostic test this bias is produced when researchers included only "clear" or "definite" cases, not representing the whole spectrum of disease presentation, and/or "clear" or healthy controls subjects, not representing the conditions in which a differential diagnosis should be carried out. Sensitivity and specificity of a diagnostic test are increased. ${ }^{16}$ A particular case is the purity diagnostic bias, when selecting cases of a certain disease those with other comorbidities are excluded and the final sample does not represent the cases originated. ${ }^{3}$

- Survivor treatment selection bias: in observational studies patients who live longer have more probability to receive a certain treatment. A retrospective analysis can therefore yield a positive association between that treatment and survival. ${ }^{17}$

In occupational studies a bad definition, albeit unavoidable, of eligible population frequently occurs. It is produced by the healthy worker effect: the lower mortality observed in the employed population when compared with the general population. Furthermore, those who remain employed tend to be healthier than those who leave employment. ${ }^{18}$

Inadequate definition of the eligible population can happen frequently in case-control studies, where several specific biases have been described regarding the selection of the reference population (controls). The most common are (in alphabetical order):

- Berkson's bias: first described by Berkson in 1946 for casecontrol studies. ${ }^{19}$ It is produced when the probability of hospitalisation of cases and controls differ, and it is also influenced by the exposure. See Feinstein et $a l^{20}$ and Flanders $e t a^{21}$ for a discussion and solution.

- Exclusion bias: when controls with conditions related to the exposure are excluded, whereas cases with these diseases as comorbidities are kept in the study. This was the explanation given for the association between reserpine and breast cancer: controls with cardiovascular disease (a common comorbidity and related to the use of reserpine) were excluded but this criterion was not applied to cases, thus yielding a spurious association between reserpine and breast cancer. ${ }^{22}$

- Friend control bias: It was assumed that the correlation in exposure status between cases and their friend controls lead to biased estimates of the association between exposure and outcome. In a matched study, with a matched analysis, there is no bias if the exposure induced risks of disease are constant over time and there are not gregarious subjects, individuals elected by more than one case. ${ }^{23}$ 
- Inclusion bias: produced in hospital based case-control studies when one or more conditions of controls are related with the exposure. The frequency of exposure is higher than expected in the reference group, producing a toward the null bias. ${ }^{1}$

- Matching: It is well known that matching, either individual or frequency matching, introduces a selection bias, which is controlled for by appropriate statistical analysis: matched analysis in studies with individual matching and adjusting for the variables used to match in frequency matching. Overmatching is produced when researchers match by a non-confounding variable (associated to the exposure but not to the disease) and can underestimate an association. $^{1}$

- Relative control bias: It was assumed that the correlation in exposure status between cases and their relative controls yield biased estimates of the association between exposure and outcome. In a matched study, with a matched analysis, there is no bias if the exposure induced risks of disease are constant over time. ${ }^{24}$

In systematic reviews and meta-analyses language bias is a kind of inappropriate definition of the eligible population (the reports studying the relevant topic). In systematic reviews and meta-analysis it has been common to exclude reports in other languages than English. Egger et $a l^{25}$ showed that there was a trend to publish in English compared with German when the results achieved statistical significance; later on, the same group found that language bias has in general little effect on summary effect estimates. ${ }^{26}$

\subsection{Lack of accuracy of sampling frame}

The most common bias in this group is non-random sampling bias: obviously, this selection procedure can yield a nonrepresentative sample in which a parameter estimate differs from the existing at the target population. ${ }^{4}$ A particular case of this bias is telephone random sampling bias: it excludes some households from the sample, thus producing a coverage bias. In the US it has been shown that the differences between participants and non-participants are generally not large, ${ }^{27}$ but the situation can be very different in less developed countries.

In systematic reviews and meta-analyses focused only in published reports the most important selection biases are publication bias and other biases influencing the identification of relevant studies (citation bias and dissemination bias):

- Citation bias: articles more frequently cited are more easily found and included in systematic reviews and metaanalysis. Citation is closely related to the impact factor of the publishing journal. ${ }^{28}$ In certain fields, citation has been related to statistical significance. ${ }^{29}$

- Dissemination bias: the biases associated to the whole publication process, from biases in the retrieval of information (including language bias) to the way the results are reported..$^{30}$

- Post hoc analysis: the fishing expeditions with data dredging originates post hoc questions and subgroup analysis with misleading results. ${ }^{4}$ Given that the reports based on post hoc analysis are frequently reported when significant results are observed, this bias is relevant for meta-analysis of published studies as a form of publication (selection) bias.

- Publication bias: regarding an association that is produced when the published reports do not represent the studies carried out on that association. Several factors have been found to influence publication, the most important being statistical significance, size of the study, funding, prestige, type of design, and study quality. ${ }^{31}$

\subsection{Uneven diagnostic procedures in the target population}

In case-control studies, if exposure influences the diagnosis of the disease, detection bias occurs. Particular types of this bias are: exposure can be taken as another diagnostic criterion (diagnostic suspicion bias). ${ }^{3}$ Exposure can trigger the search for the disease; for instance, benign anal lesions increases the diagnosis of anal cancer..$^{32}$ Exposure may produce a symptom/ sign that favours diagnosis (unmasking-detection signal-bias) ${ }^{3}$ or a benign condition close clinically to the disease (mimicry bias). ${ }^{3}$ In other designs (such as cohort studies) detection bias is an information bias.

\subsection{During study implementation}

The three most common biases at this stage are losses to follow up, missing information in multivariable analysis, and non-response bias:

- Losses/withdrawals to follow up: in both cohort and experimental studies when losses/withdrawals are uneven in both the exposure and outcome categories, the validity of the statistical results may be affected. ${ }^{33}$

- Missing information in multivariable analysis: multivariable analysis selects records with complete information on the variables included in the model. If participants with complete information do not represent the target population, it can introduce a selection bias. ${ }^{7}$ This bias is relevant in studies, mainly retrospective, using data from the clinical chart, in which patients with more complete data have more severe diseases or stay longer at hospital, or both.

- Non-response bias: when participants differ from nonparticipants, for example, Melton et al. ${ }^{34}$ The healthy volunteer effect is a particular case: when the participants are healthier than the general population. ${ }^{35}$ This is particularly relevant when a diagnostic manoeuvre, such as a screening test, is evaluated in the general population, producing an away from the null bias; thus the benefit of the intervention is spuriously increased.

\section{INFORMATION BIAS}

Information bias occurs during data collection. The three main types of information bias are misclassification bias, ecological fallacy, and regression to the mean. Other information biases are also described.

\subsection{Misclassification bias}

It is originated when sensitivity and/or specificity of the procedure to detect exposure and/or effect is not perfect, that is, exposed/diseased subjects can be classified as nonexposed/non-diseased and vice versa. ${ }^{36}$ Given that perfect tools to gather data are very uncommon most studies must assume a certain degree of misclassification. Random error also can produce it. ${ }^{37}$ This implies that random errors in data entry/capture, missing data, end digit preference (rounding to 5 or 0 ), frequently unavoidable, also introduce misclassification. There are two major types of misclassification bias:

- Differential misclassification bias: when misclassification is different in the groups to be compared; for example, in a case-control study the recalled exposure is not the same for cases and controls. The estimate is biased in either direction, toward the null or away from the null. ${ }^{36}$

- Non-differential misclassification bias: when the misclassification is the same across the groups to be compared, for 
example, exposure is equally misclassified in cases and controls. For binary variables the estimate is biased toward the null value ${ }^{36}$; however, for variables with more than two categories (polytomous) this rule may not hold and an away from the null bias can be obtained. ${ }^{38}$

The most common biases producing misclassification are the following

- Detection bias in studies with follow up (cohorts, clinical trials) is an information bias.

- Observer/interviewer bias: the knowledge of the hypothesis, the disease status, or the exposure status (including the intervention received) can influence data recording (observer expectation bias). ${ }^{3}$ The means by which interviewers can introduce error into a questionnaire include administering the interview or helping the respondents in different ways (even with gestures), putting emphases in different questions, and so on. ${ }^{39}$ A particular situation is when the measure of an exposure influences its value (for example, blood pressure) (apprehension bias). ${ }^{3}$

- Recall bias: if the presence of disease influences the perception of its causes (rumination bias) ${ }^{3}$ or the search for exposure to the putative cause (exposure suspicion bias), ${ }^{3}$ or in a trial if the patient knows what they receive may influence their answers (participant expectation bias). ${ }^{3}$ This bias is more common in case-control studies, in which participants know their diseases, although it can occur in cohort studies (for example, workers who known their exposure to hazardous substances may show a trend to report more the effects related to them), and trials without participants' blinding. ${ }^{40}$

- Reporting bias: participants can "collaborate" with researchers and give answers in the direction they perceive are of interest (obsequiousness bias), ${ }^{3}$ or the existence of a case triggers family information (family aggregation bias), see Khoury et $a l^{41}$ for an example. Measures or sensitive questions that embarrass or hurt can be refused (unacceptable disease/exposure). ${ }^{3}$ Underreporting bias is common with socially undesirable behaviours, such as alcohol consumption. ${ }^{42}$ The mode for mean bias occurs when frequency-quantity questionnaires are used to assess consumption of alcohol and foods, subjects tend to report modal rather than average behaviour, hence with data skewed towards zero, the average intakes are underestimated, leading to overestimation of the gradient with risk. $^{43}$

The last three biases can be reduced using blinding, a procedure by which subjects ignore some important aspects of a research to avoid differential misclassification bias. In trials, blinding means that participants do not know the intervention they receive (participants blinding) and/or observers do not know the intervention received by participants (observer blinding), and/or data analysts do not know the labels of the groups to be compared..$^{44}$ In observational research, observer blinding can rarely be applied with the same goal of trials, but more frequently observers and participants are blind to the main hypotheses (and related questions) of a research.

\subsection{Ecological fallacy}

It is a bias produced when analyses realised in an ecological (group level) analysis are used to make inferences at the individual level. For instance, if exposure and disease are measured at the group level (for example, exposure prevalence and disease risk in each country), exposure-disease relations can be biased from those obtained at the individual level (for example, exposure status and disease status in each subject). Ecological fallacy can be produced by within group (individual level) biases, such as confounding, selection bias, or misclassification, and by confounding by group or effect modification by group. ${ }^{45}$ Effect modification by group on an additive scale is produced when the rate difference for the exposure effect changes across communities ${ }^{45}$; for example, lets suppose three groups with exposure prevalences of 35\%, $50 \%$, and $65 \%$, a similar rate of disease in the non-exposed of $100 / 100000$, and rates of disease in the exposed of 286, 200, and 154/100 000. In this example, the rate difference in each group is positive (the exposure increases the risk of the disease), although an ecological analysis do find a negative relation.

\subsection{Regression to the mean}

It is the phenomenon that a variable that shows an extreme value on its first assessment will tend to be closer to the centre of its distribution on a later measurement. ${ }^{46}$ This bias is relevant when the efficacy of a treatment to reduce high levels of a variable (for example, cholesterol) is assessed, when researchers are interested in the relation between the initial value of a variable and the change in that measurement over time, or when two methods of measurement are compared.$^{47}$ The two usual ways of neutralising this bias are with the existence of an appropriate reference group and a selection based on more than one measurement.

The regression dilution bias is related to the regression to the mean. This bias is produced in longitudinal studies analysing baseline determinations of a continuous variable (such as diastolic blood pressure (DBP)) to an outcome (for example, stroke). Baseline DBP measurements randomly fluctuates among individuals because of two reasons: variations in the measurement process and temporary deviations at the baseline determination from the usual DBP level. This underestimates the real association between exposure and outcome because extreme categories include more people than they should, that is, the bottom category of baseline DBP has more people whose DBP level is somewhat lower than their usual DBP, whereas the top category of DBP includes more subjects with higher baseline DBP than their usual DBP level. ${ }^{48}$

\subsection{Other information biases}

- Hawthorne effect: described in the 1920s in the Hawthorne plant of the Western Electric Company (Chicago, IL). It is an increase in productivity-or other outcome under study -in participants who are aware of being observed. ${ }^{49}$ For example, laboratory physicians increase their agreement rate after knowing that they participate in a research on reliability of diagnostic tests. ${ }^{50}$

- Lead time bias: the added time of illness produced by the diagnosis of a condition during its latency period. This bias is relevant in the evaluation of the efficacy of screening, in which the cases detected in the screened group has a longer duration of disease than those diagnosed in the non-screened one. ${ }^{51}$

- Protopathic bias: when a exposure is influenced by early (subclinical) stages of disease. For instance, preclinical pancreatic cancer can produce diabetes mellitus, and thus an association between diabetes and cancer can occur. ${ }^{52}$ It is also produced when a pharmaceutical agent is prescribed for an early manifestation of a disease that has not been yet diagnosed. ${ }^{53}$ The sick quitter bias is related to protopathic bias: people with risky behaviours (such as heavy alcohol consumption) quit their habit as a consequence of disease ${ }^{54}$; studies analysing current behaviour as a risk factor will labelled them as non-exposed, thus underestimating the true association. 
- Temporal ambiguity: when it cannot be established that exposure precedes effect. It is common in cross sectional and ecological studies. ${ }^{1}$

- Will Rogers phenomenon: named in honour of the philosopher Will Rogers by Feinstein et al. ${ }^{55}$ The improvement in diagnostic tests refines disease staging in diseases such as cancer. This produces a stage migration from early to more advances stages and an apparent higher survival. This bias is relevant when comparing cancer survival rates across time or even among centres with different diagnostic capabilities (for example, tertiary compared with primary care hospitals).

- Work up bias (verification bias): in the assessment of validity of a diagnostic test, it is produced when the execution of the gold standard is influenced by the results of the assessed test, typically the reference test is less frequently performed when the test result is negative. ${ }^{166}$ This bias is aggravated when the clinical characteristics of a disease influence in the test results. ${ }^{57}$

\section{CONFOUNDING}

It occurs when a variable is a risk factor for an effect among non-exposed persons and is associated with the exposure of interest in the population from which the effect derives, without being affected by the exposure or the disease (in particular, without being an intermediate step in the causal pathway between the exposure and the effect). ${ }^{1}$ The counterfactual approach is the current procedure to explain confounding adequately, ${ }^{58}$ and causal diagrams help to identify it. ${ }^{59}$ Confounding can occur in every epidemiological study. Susceptibility bias is a synonym: when people who are particularly susceptible to development of a outcome are also prone to be exposed; for example, women with threatened abortion have a high probability of delivering a malformed fetus but also have a high probability of receiving hormone treatment. This can yield a spurious association between hormones and congenital malformations. ${ }^{53}$

Confounding can be neutralised at the design stage of a research (for example, by matching or randomisation) and/or at the analysis, given that the confounders have been measured properly. Misclassification of confounders hinders their control in analysis. Non-differential misclassification of a binary confounder reduces the ability of analysis to control for the confounder, ${ }^{60}$ whereas this in a polytomous confounder can produce estimates biased in either direction. ${ }^{61}$ Particular types of confounding bias are the following:

- Confounding by group: it is produced in an ecological study, when the exposure prevalence of each community (group) is correlated with the disease risk in non-exposed of the same community. It can be a mechanism for producing ecological fallacy. ${ }^{45}$ For example, lets suppose three communities (A, B, C) with prevalence exposures of $10 \%, 20 \%$, and $30 \%$, rates of disease in non-exposed of $2 \%$, $3 \%$, and $4 \%$, and rates of disease in the exposed of $2 \%, 3 \%$, $4 \%$, respectively. There is no association between the exposure and the disease as the three relative risks are one, although an ecological analysis, regressing the rate of disease on the prevalence of exposure, does find a positive association.

- Confounding by indication: this is produced when an intervention (treatment) is indicated by a perceived high risk, poor prognosis, or simply some symptoms. Here the confounder is the indication, as it is related to the intervention and is a risk indicator for the disease. ${ }^{62}$ For example, in the study of the association between cimetidine and gastric cancer, the indication peptic ulcer is considered the potential confounder. ${ }^{63}$ This kind of bias occurs in observational studies (mainly retrospective) analysing interventions. Sometimes confounding by indication is mistaken for protopathic bias.

\section{SPECIFIC BIASES IN TRIALS}

- Allocation of intervention bias: when intervention is differentially assigned to the population. It is more common in non-randomised trials. In randomised trials it is recommended concealment of the allocation sequence of intervention. ${ }^{64}$ If the sequence is known in advance may produce selection bias. It has been shown that trials in which concealment was unclear or inadequate, compared with trials with adequate concealment, report larger estimates of treatment effects. ${ }^{65}$

- Compliance bias: in trials requiring adherence to intervention, the degree of adherence (compliance) influences efficacy assessment of the intervention. For example, when high risk patients quit exercise programmes. ${ }^{3}$

- Contamination bias: when intervention-like activities find their way into the control group. It biases the estimate of the intervention effect toward the null hypothesis. ${ }^{66}$ It occurs more frequently in community intervention trials because of the relationships among members of different communities and interference by mass media, health professionals, etc.

- Differential maturing: in group randomised trials differential maturing reflects uneven secular trends among the groups in the trial favouring one condition or another. ${ }^{66}$

- Lack of intention to treat analysis: in randomised studies the analysis should be done keeping participants in the group they were assigned to. The goals of randomisation are to avoid confounding and selection bias. If non-compliant participants or those receiving a wrong intervention are excluded from the analysis, the branches of a randomised trial may not be comparable. There are exceptions to the rule of intention to treat analysis. ${ }^{67}$

\section{Authors' affiliations}

M Delgado-Rodríguez, Division of Preventive Medicine and Public Health, University of Jaen, Spain

J Llorca, Department of Preventive Medicine and Public Health, University of Cantabria, Spain

\section{REFERENCES}

1 Rothman K, Greenland S. Modern epidemiology. 2nd ed. Boston: LippiconttRaven, 1998.

2 Kleinbaum DG, Kupper LL, Morgenstern H. Epidemiologic research. Belmont, CA: Lifetime Learning Publications, 1982.

3 Sackett DL. Bias in analytic research. J Chron Dis 1979;32:51-63.

4 Choi BCK. Bias, overview. In: Gail MH, Benichou J, eds. Encyclopedia of epidemiologic methods. Chichester: Wiley, 2000:74-82.

5 Maclure M, Schneeweiss S. Causation of bias: the episcope. Epidemiology $2001 ; 12: 114-22$.

6 Steineck G, Ahlbom A. A definition of bias founded on the concept of the study base. Epidemiology 1992;3:477-82.

7 Ellenberg JH. Selection bias in observational and experimental studies. Stat Med 1994; 13:557-67.

8 Kleinbaum DG, Morgenstern H, Kupper LL. Selection bias in epidemiologic studies. Am J Epidemiol 1981;113:452-63.

9 Greenland S. Basic methods for sensitivity analysis of biases. Int J Epidemiol 1996;25:1107-16.

10 Pollock AM, Benster R, Vickers N. Why did treatments rates for colorectal cancer in south east England fall between 1982 and 1988 ? The effect of case ascertainment and registration bias. J Public Health Med 1995;17:419-28.

11 Chiang CL. Competing risks in mortality analysis. Annu Rev Public Health $1991 ; 12: 281-307$.

12 Gail M. Competing risks. In: Kotz S, Johnson NL, eds. Encyclopedia of statistical sciences. Vol 2. New York: Wiley, 1982:75-81.

13 Simon R. Length biased sampling in etiologic studies. Am J Epidemiol 1980;111:444-52.

14 Neyman J. Statistics: servant of all sciences. Science 1955;122:401-6. 
15 Hill G, Connelly J, Hébert R, et al. Neyman's bias re-visited. J Clin Epidemiol 2003;56:293-6.

16 Ransohoff DF, Feinstein AR. Problems of spectrum and bias in evaluating the efficacy of diagnostic tests. N Engl J Med 1978;299:926-30.

17 Glesby MJ, Hoover DR. Survivor treatment selection bias in observational studies: examples from the AIDS literature. Ann Intern Med 1996; 124:999-1005

18 Arrighi HM, Hertz-Picciotto I. The evolving concept of the healthy worker survivor effect. Epidemiology 1994;5:189-96.

19 Berkson J. Limitations of the application of fourfold table analysis to hospital data. Biomet Bull 1946;2:47-53.

20 Feinstein AR, Walter SD, Horwitz RI. An analysis of Berkson's bias in casecontrol studies. J Chron Dis 1986;39:495-504.

21 Flanders WD, Boyle CA, Boring JR. Bias associated with differential hospitalization rates in incident case-control studies. J Clin Epidemiol 1989;42:395-401.

22 Horwitz RI, Feinstein AR. Exclusion bias and the false relationship of reserpine and breast cancer. Arch Intern Med 1985;145:1873-5.

23 Robins JM, Pike M. The validity of case-control studies with nonrandom selection of controls. Epidemiology 1990;1:273-84.

24 Goldstein AM, Hodge SE, Haile RWC. Selection bias in case-control studies using relatives as controls. Int J Epidemiol 1989;18:985-9.

25 Egger M, Zellweger-Zähner, Schneider M, et al. Language bias in randomised controlled trials published in English and German. Lancet 1997;350:326-9.

26 Jüni P, Holenstein F, Sterne J, et al. Direction and impact of language bias in meta-analyses of controlled trials: empirical study. Int J Epidemiol 2002;31:115-23.

27 Brick JM, Waksberg J, Kulp D, et al. Bias in list-assisted telephone samples. Public Opinion Quarterly 1995:59:218-35.

28 Callagham M, Wears RL, Weber E. Journal prestige, publication bias, and other characteristics associated with citation of published studies in peerreviewed journals. JAMA 2002;287:2847-50.

29 Kjaergard LL, Gluud C. Citation bias of hepato-biliary randomized clinical trials. J Clin Epidemiol 2002;55:407-10.

30 Song F, Easterwood A, Gilbody S, et al. Publication and related biases. Health Technol Assess 2000;4:1-115.

31 Sterne JAC, Gavaghan D, Egger M. Publication and related bias in meta analysis: power of statistical tests and prevalence in the literature. $J$ Clin Epidemiol 2000;53:1 119-29.

32 Frisch $M$, Olsen JH, Bautz A, et al. Benign anal lesions and the risk of anal cancer. N Engl J Med 1994;331:300-2.

33 Greenland S. Response and follow-up bias in cohort studies. Am J Epidemiol 1977; 106:184-7.

34 Melton JL III , Dyck PJ, Karnes JL, et al. Non-response bias in studies of diabetic complications: the Rochester diabetic neuropathy study. J Clin Epidemiol 1993;46:341-8

35 Froom P, Melamed S, Kristal-Boneh E, et al. Healthy volunteer effect in industrial workers. J Clin Epidemiol 1999:52:731-5.

36 Copeland KT, Checkoway H, McMichael AJ, et al. Bias due to misclassification in the estimation of relative risk. Am J Epidemio 1977; 105:488-95.

37 Brenner $\mathbf{H}$, Blettner M. Misclassification bias arising from random error in exposure measurement: implications for dual measurement strategies. Am J Epidemiol 1993;138:453-61.

38 Dosemeci M, Wacholder S, Lubin JH. Does nondifferential misclassification of exposure always bias a true effect toward the null value. Am J Epidemiol $1990 ; 132: 746-8$

39 Johannes CB, Crawford SL, McKinlay JB. Interviewer effects in a cohort study. Results from the Massachusetts women's health study. Am J Epidemiol 1997; 146:429-38.

40 Neugebaver $\mathrm{R}, \mathrm{Ng} \mathrm{S}$. Differential recall as a source of bias in epidemiologic research. J Clin Epidemiol 1990;43:1337-41.

41 Khoury MJ, Flanders WD. Bias in using family history as a risk factor in casecontrol studies of disease. Epidemiology 1995;6:511-19.
42 Wynder EL. Investigator bias and interviewer bias: the problem of systematic error in epidemiology. J Clin Epidemiol 1994;47:825-7.

43 Willet W. Nutritional epidemiology. New York: Oxford University Press, 1989.

44 Schulz KF, Grimes DA. Blinding in randomised trials: hiding who got what. Lancet 2002;359:696-700.

45 Morgenstern H. Ecologic study. In: Gail MH, Benichou J, eds. Encyclopedia of epidemiologic methods. New York: Wiley, 2000:315-36.

46 Davis CE. The effect of regression to the mean in epidemiologic and clinical studies. Am J Epidemiol 1976;104:493-8.

47 Bland JM, Altman DG. Some examples of regression towards the mean. BMJ 1994:309:780.

48 MacMahon S, Peto R, Cutler J, et al. Blood pressure, stroke, and coronary heart disease. Part 1, prolonged differences in blood pressure: prospective observational studies corrected for the regression dilution bias. Lancet 1990;335:765-74.

49 Wickström G, Bendix T. The "Hawthorne effect" - what did the original Hawthorne studies actually show? Scand J Work Environ Health 2000;26:363-7.

50 Hernández Aguado I, Ruiz Atienza L, Fernández García I, et al. Evaluación de la variabilidad del diagnóstico rápido de infección por Chlamydia tracomatis mediante inmunofluorescencia directa. Med Clín (Barc) 1990;94:9-11

51 Fletcher RH, Fletcher SW, Wagner EH. Clinical epidemiology. The essentials. 2nd ed. Baltimore: Williams and Wilkins, 1988:161.

52 Gullo L, Pezzilli R, Morselli-Labate AM and the Italian Pancreatic Cancer Study Group. Diabetes and the risk of pancreatic cancer. N Engl J Med 1994:331:81-4.

53 Feinstein AR, Horwitz RI. An algebraic analysis of biases due to exclusion, susceptibility, and protophatic prescription in case-control research. J Chron Dis 1981;34:393-403.

54 Shaper AG, Walker M, Wannamethee G. Alcohol and mortality in British men: explaining the U-shaped curve. Lancet 1988;ii:1267-73.

55 Feinstein AR, Sosin DM, Wells CK. The Will Rogers phenomenon. Stage migration and new diagnostic techniques as a source of misleading statistics for survival in cancer. N Engl J Med 1985;312:1604-8.

56 Zhou XH. Correcting for verification bias in studies of a diagnostics test's accuracy. Stat Method Med Res 1998;7:337-53.

57 Knottnerus JA, Leffers $P$. The influence of referral patterns on the characteristics of diagnostic tests. J Clin Epidemiol 1992;45:1143-54.

58 Maldonado G, Greenland S. Estimating causal effects. Int J Epidemiol 2002;31:422-9.

59 Greenland S, Brumback B. An overview of relations among causal modelling methods. Int J Epidemiol 2002;31:1030-7.

60 Greenland S. The effect of misclassification in the presence of covariates. Am J Epidemiol 1980;1 12:564-9.

61 Brenner H. Bias due to non-differential misclassification of polytomous confounders. J Clin Epidemiol 1993;46:57-63.

62 Salas M, Hofman A, Stricker BHC. Confounding by indication: an example of variation in the use of epidemiologic terminology. Am J Epidemiol 1999; 149:981-3

63 Walker AM. Confounding by indication. Epidemiology 1996;7:335-6.

64 Moher D, Schulz KF, Altman DG, for the CONSORT Group. The CONSORT statement: revised recommendations for improving the quality of reports of parallel-group randomised trials. Lancet 2001;357:1191-4.

65 Schulz KF, Chalmers I, Hayes RJ, et al. Empirical evidence of bias. Dimensions of methodological quality associated with estimates of treatment effects in controlled trials. JAMA 1995;273:408-12.

66 Murray DM. Statistical models appropriate for designs often used in grouprandomized trials. Stat Med 2001 20:1373-85.

67 Lewis JA, Machin D. Intention to treat-who should use ITT? Br J Cancer 1993;68:647-50 